\title{
FAN'S INEQUALITY IN THE CONTEXT OF $M_{p}$-CONVEXITY
}

\author{
CONSTANTIN P. NICULESCU AND IONEL ROVENTA
}

Abstract. Several analogues of Fan's inequality are proved in the context of $M_{p}$-convexity. As a consequence, a Nash equilibrium theorem is obtained.

\section{INTRODUCTION}

In what follows we are interested in a class of functions having a nice behavior under the action of means.

The weighted $M_{p}$-mean is defined for pairs of positive numbers $x, y$ by the formula

$$
M_{p}(x, y, 1-\lambda, \lambda)=\left\{\begin{array}{cl}
\left((1-\lambda) x^{p}+\lambda y^{p}\right)^{1 / p}, & \text { if } p \in \mathbb{R} \backslash\{0\} \\
x^{1-\lambda} y^{\lambda}, & \text { if } p=0 \\
\min \{x, y\}, & \text { if } p=-\infty \\
\max \{x, y\}, & \text { if } p=\infty,
\end{array}\right.
$$

where $\lambda \in[0,1]$. If $p$ is an odd number, we can extend $M_{p}$ to pairs of real numbers.

Let $E$ be a linear topological space and assume that $C$ is a nonempty compact and convex subset of $E$.

Definition 1. We say that a function $f: C \longrightarrow \mathbb{R}$ is $M_{p}$-concave if

$$
f((1-\lambda) x+\lambda y) \geq M_{p}(f(x), f(y), 1-\lambda, \lambda)
$$

for all $x, y \in C$ and $\lambda \in(0,1)$.

Thus the $M_{1}$-concave functions are the usual concave functions, while the $M_{\infty}$ concave functions, are precisely the quasi-concave functions.

A celebrated result due to Ky Fan asserts that any function $f: C \times C \rightarrow \mathbb{R}_{+}$ which is quasi-concave in the first variable and lower semicontinuous in the second variable verifies the inequality

$$
\min _{y \in C} \sup _{x \in C} f(x, y) \leq \sup _{z \in C} f(z, z) .
$$

The aim of this paper is to prove a complementary result, precisely:

Theorem 1. Suppose that $f: C \times C \rightarrow \mathbb{R}_{+}$is a function which is $M_{p}$-concave and lower-semicontinuous in each variable. Then

$$
\min _{y \in C} \sup _{x \in C} M_{p}^{p}(f(x, y), f(y, x), 1-\lambda, \lambda) \leq \sup _{z \in C} f^{p}(z, z),
$$

for all $\lambda \in(0,1)$ and $p \in \mathbb{R}$.

2000 Mathematics Subject Classification. Primary 05C38, 15A15; Secondary 05A15, 15A18.

Key words and phrases. $M_{p^{-}}$convexity, min-max inequality, Nash equilibrium.

Published in vol.: Applied Analysis and Differential Equations. Proc. ICAADE 2006, pp. 267-274, World Scientific, Singapore, 2007 (Editors, Ovidiu Carja and Ioan I. Vrabie). ISBN 978-981-270-594-5, ISSN 981-270-594-5. Revised, February 12, 2009. 
Our technics yields also the following fact:

Theorem 2. Let $C$ be a nonempty compact and convex subset of $E$, and let $f$ : $C \times C \rightarrow \mathbb{R}_{+}$be a function which is $M_{p}$-concave in the first variable and lowersemicontinuous in the second variable. Then

$$
\min _{y \in C} \max _{x \in C} M_{p}^{p}(f(y, y), f(x, x), 1-\lambda, \lambda) \leq \sup _{z \in C} f^{p}(z, z)
$$

for all $\lambda \in(0,1)$ and $p \in \mathbb{R}$.

For $p$ an odd number $f$ is allowed to take negative values.

\section{Proof of the main Result}

We actually prove a much more general result:

Theorem 3. Assume $f: C \times C \rightarrow \mathbb{R}_{+}$is a function which is $M_{p}$-concave and lower-semicontinuous in each variable and let $g: C \rightarrow C$ be a continuous onto function. Then

$$
\min _{y \in C} \sup _{x \in C} M_{p}^{p}(f(x, y), f(y, x), 1-\lambda, \lambda) \leq \sup _{z \in C} M_{p}^{p}(f(z, g(z)), f(g(z), z), 1-\lambda, \lambda),
$$

for all $\lambda \in(0,1)$ and $p \in \mathbb{R}$.

Theorem 1 represents the particular case where $g$ is the identity of $C$.

The proof of Theorem 3 is based on the KKM-Theorem, whose statement is recalled here for the convenience of the reader:

Theorem 4. (Knaster-Kuratowski-Mazurkievicz). Suppose that for every point $x$ in a nonempty set $X \subset E$ there is an associated closed subset $M(x) \subset X$ such that

$$
\operatorname{conv} F \subset \bigcup_{x \in F} M(x)
$$

holds for all finite subsets $F \subset X$. Then for any finite subset $F \subset X$ we have

$$
\bigcap_{x \in F} M(x) \neq \emptyset \text {. }
$$

Hence if some subset $M(z)$ is compact, we have

$$
\bigcap_{x \in X} M(x) \neq \emptyset .
$$

Theorem 4 is one of the many results known to be equivalent to Brouwer's fixed point theorem. See [1].

Proof of Theorem 3. We attach to $g: C \rightarrow C$ and $\lambda \in[0,1]$ the family of sets $(M(x))_{x \in C}$, where $M(x)$ consists of all $y \in C$ such that

$$
M_{p}^{p}(f(x, y), f(y, x), 1-\lambda, \lambda) \leq \sup _{z \in C} M_{p}^{p}(f(z, g(z)), f(g(z), z), 1-\lambda, \lambda) .
$$

We will show that this family satisfies the hypothesis of the KKM-Theorem. In fact, $g(x) \in M(g(x))$ for every $x \in C$ and

$$
\operatorname{conv} F \subset \bigcup_{x \in F} M(g(x))
$$


for every finite subset $F \subset C$. For example, if $F$ consists of two elements $g\left(x_{1}\right)$ and $g\left(x_{2}\right)$, we have to show that

$$
u=(1-\alpha) g\left(x_{1}\right)+\alpha g\left(x_{2}\right) \in M\left(g\left(x_{1}\right)\right) \cup M\left(g\left(x_{2}\right)\right)
$$

for every $\alpha \in(0,1)$. Our argument is by reductio ad absurdum.

If (2.1) fails, then for some $\alpha \in(0,1)$ we have

$$
M_{p}^{p}\left(f\left(x_{1}, u\right), f\left(u, x_{1}\right), 1-\lambda, \lambda\right)>\sup _{z \in C} M_{p}^{p}(f(z, g(z)), f(g(z), z), 1-\lambda, \lambda),
$$

and

$$
M_{p}^{p}\left(f\left(x_{2}, u\right), f\left(u, x_{2}\right), 1-\lambda, \lambda\right)>\sup _{z \in C} M_{p}^{p}(f(z, g(z)), f(g(z), z), 1-\lambda, \lambda) .
$$

The Intermediate Value Theorem yields a $\beta \in[0,1]$ such that $x_{\beta}=\beta x_{1}+(1-$ $\beta) x_{2} \in C$ verifies

$$
g\left(x_{\beta}\right)=u=(1-\alpha) g\left(x_{1}\right)+\alpha g\left(x_{2}\right) .
$$

Since $f$ is $M_{p}$-concave in each variable it follows that

$$
\begin{aligned}
M_{p}^{p}\left(f\left(x_{\beta}, g\left(x_{\beta}\right)\right), f\right. & \left.\left(g\left(x_{\beta}\right), x_{\beta}\right), 1-\lambda, \lambda\right) \\
& =(1-\lambda) f^{p}\left((1-\beta) x_{1}+\beta x_{2}, u\right)+\lambda f^{p}\left(u,(1-\beta) x_{1}+\beta x_{2}\right)
\end{aligned}
$$

is not less than

$$
\begin{gathered}
(1-\lambda)\left((1-\beta) f^{p}\left(x_{1}, u\right)+\beta f^{p}\left(x_{2}, u\right)\right)+\lambda\left((1-\beta) f^{p}\left(u, x_{1}\right)+\beta f^{p}\left(u, x_{2}\right)\right) \\
=(1-\beta)\left((1-\lambda) f^{p}\left(x_{1}, u\right)+\lambda f^{p}\left(u, x_{1}\right)\right)+\beta\left((1-\lambda) f^{p}\left(x_{2}, u\right)+\lambda f^{p}\left(u, x_{2}\right)\right) \\
=(1-\beta) M_{p}^{p}\left(f\left(x_{1}, u\right), f\left(u, x_{1}\right), 1-\lambda, \lambda\right)+\beta M_{p}^{p}\left(f\left(x_{2}, u\right), f\left(u, x_{2}\right), 1-\lambda, \lambda\right) \\
>(1-\beta) \sup _{z \in C} M_{p}^{p}(f(z, g(z)), f(g(z), z), 1-\lambda, \lambda) \\
+\beta \sup _{z \in C} M_{p}^{p}(f(z, g(z)), f(g(z), z), 1-\lambda, \lambda) \\
=\sup _{z \in C} M_{p}^{p}(f(z, g(z)), f(g(z), z), 1-\lambda, \lambda),
\end{gathered}
$$

a contradiction. Thus (2.1) follows.

By the KKM-Theorem we infer that

$$
\bigcap_{x \in C} M(g(x)) \neq \emptyset
$$

which means the existence of $y \in C$ such that

$$
M_{p}^{p}(f(x, y), f(y, x), 1-\lambda, \lambda) \leq \sup _{z \in C} M_{p}^{p}(f(z, g(z)), f(g(z), z), 1-\lambda, \lambda),
$$

for every $x \in C$, equivalently,

$$
\sup _{x \in C} M_{p}^{p}(f(x, y), f(y, x), 1-\lambda, \lambda) \leq \sup _{z \in C} M_{p}^{p}(f(z, g(z)), f(g(z), z), 1-\lambda, \lambda) .
$$

In conclusion,

$$
\min _{y \in C} \sup _{x} M_{p}^{p}(f(x, y), f(y, x), 1-\lambda, \lambda) \leq \sup _{z \in C} M_{p}^{p}(f(z, g(z)), f(g(z), z), 1-\lambda, \lambda) .
$$




\section{A nonsymmetric extension of Fan's Theorem}

The aim of this section is to prove the following result:

Theorem 5. Let $D$ and $F$ be two two nonempty, compact and convex subsets of $E$ and let $g$ be a continuous onto function $g: D \rightarrow F$. Then for every function $f$ : $D \times F \rightarrow \mathbb{R}_{+}$which is quasi-concave in the first variable and lower-semicontinuous in the second variable the following inequality holds:

$$
\min _{y \in F} \sup _{x \in D} f(x, y) \leq \sup _{z \in D} f(z, g(z)) .
$$

This result extends Fan's Theorem (which corresponds to the case where $D=F$ and $g(z)=z$ for all $z \in D$ ).

Proof. Consider the family of sets

$$
M(g(x))=\left\{y \in F: f(x, y) \leq \sup _{z \in D} f(z, g(z))\right\}, \quad \text { for } x \in D .
$$

We will show that this family verifies the assumptions of the KKM-Theorem. In fact, it is easy to see that $g(x) \in M(g(x))$, for $x \in D$. Let $A$ be a nonempty finite subset of $F$, say $A=\left\{g\left(x_{1}\right), g\left(x_{2}\right)\right\}$ for simplicity. We have to prove that

$$
\operatorname{conv} A \subset \bigcup_{g(x) \in A} M(g(x))
$$

that is,

$$
(1-\lambda) g\left(x_{1}\right)+\lambda g\left(x_{2}\right) \in M\left(g\left(x_{1}\right)\right) \cup M\left(g\left(x_{2}\right)\right),
$$

for all $\lambda \in(0,1)$.

Indeed, if the contrary is true, then for a suitable $\lambda \in(0,1)$ we have

$$
f\left(x_{1},(1-\lambda) g\left(x_{1}\right)+\lambda g\left(x_{2}\right)\right)>\sup _{z \in D} f(z, g(z))
$$

and

$$
f\left(x_{2},(1-\lambda) g\left(x_{1}\right)+\lambda g\left(x_{2}\right)\right)>\sup _{z \in D} f(z, g(z)) .
$$

The intermediate Value Theorem yields an $\alpha \in[0,1]$ such that $x_{\lambda}=\alpha x_{1}+(1-$ $\alpha) x_{2} \in D$ verifies

$$
(1-\lambda) g\left(x_{1}\right)+\lambda g\left(x_{2}\right)=g\left(x_{\lambda}\right)
$$

Then

$$
\begin{aligned}
f\left(x_{\lambda}, g\left(x_{\lambda}\right)\right) & =f\left(\alpha x_{1}+(1-\alpha) x_{2},(1-\lambda) g\left(x_{1}\right)+\lambda g\left(x_{2}\right)\right) \\
& \geq \min \left\{f\left(x_{1},(1-\lambda) g\left(x_{1}\right)+\lambda g\left(x_{2}\right)\right), f\left(x_{2},(1-\lambda) g\left(x_{1}\right)+\lambda g\left(x_{2}\right)\right)\right\} \\
& >\sup _{z \in D} f(z, g(z)),
\end{aligned}
$$

a contradiction that shows that (3.1) works. By the KKM-Theorem, $\cap_{x \in D} M(g(x)) \neq$ $\emptyset$, and this fact assures the existence of a $y_{0} \in F$ such that

$$
f\left(x, y_{0}\right) \leq \sup _{z \in D} f(z, g(z)) \quad \text { for every } x \in D .
$$

Consequently $\sup _{x \in D} f\left(x, y_{0}\right) \leq \sup _{z \in D} f(z, g(z))$, which yields

$$
\min _{y \in F} \sup _{x \in D} f\left(x, y_{0}\right) \leq \sup _{z \in D} f(z, g(z)) \text {. }
$$

A similar argument yields the following theorem in the case of $M_{p}$-convex functions: 
Theorem 6. Let $C$ be a nonempty, compact and convex subset of $E$ and let $g$ be a continuous onto function $g: C \rightarrow C$. Then for every $M_{p}$-convex function $f: C \times C \rightarrow \mathbb{R}_{+}$which is upper-semicontinuous with respect to each variable we have

$$
\max _{x \in C} \inf _{y \in C} M_{p}^{p}(f(x, y), f(y, x), 1-\lambda, \lambda) \geq \inf _{z \in C} M_{p}^{p}(f(z, g(z)), f(g(z), z), 1-\lambda, \lambda),
$$

for all $\lambda \in(0,1)$.

In the same manner we can prove the following result for the quasi-convex functions:

Theorem 7. Let $D$ and $F$ be two nonempty, compact and convex subsets and let $g$ be a continuous onto function $g: D \rightarrow F$. Let $f: D \times F \rightarrow \mathbb{R}_{+}$be a quasi-convex function in the second variable and upper-semicontinuous in the first variable. Then we have

$$
\max _{x \in D} \inf _{y \in F} f(x, y) \geq \inf _{z \in D} f(z, g(z)) .
$$

Remark 1. We impose the condition that $g$ is an onto function in order to assure the applicability of KKM-Theorem.

\section{Further Results}

An important application of Theorem 5 is the existence of a $g$-equilibrium, a fact that generalizes the existence of a Nash equilibrium.

Theorem 8. ${ }^{1}$ Let $C=C_{1} \times C_{2} \times \ldots \times C_{n}$, where $C_{i}, \quad i=1, \ldots, n$ are nonempty, compact and convex subsets of $E$, let $g=\left(g_{1}, g_{2}, \ldots, g_{n}\right): C \rightarrow C$ be a continuous onto function and let $f_{1}, \ldots, f_{n}: C \rightarrow C$ be lower-semicontinuous functions such that $x \rightarrow \sum_{i=1}^{n} f_{i}\left(y_{1}, \ldots, g\left(x_{i}\right), \ldots y_{n}\right)$ is quasi convex for each $y \in C$. Then there exists an $y \in C$ such that

$$
\left.f_{i}(y) \leq f_{i}\left(y_{1}, \ldots, g\left(x_{i}\right), \ldots, y_{n}\right)\right)
$$

for every $x_{i} \in C_{i}, i=1, \ldots, n$.

Proof. Let $f(x, y)=\sum_{i=1}^{n}\left(f_{i}(y)-f_{i}\left(y_{1}, \ldots, g\left(x_{i}\right), \ldots, y_{n}\right)\right)$. It is easy to see that $f$ satisfies the assumptions of Theorem 5 . This yields an $y \in C$ such that

$$
\sup _{x \in C} f(x, y) \leq \sup _{z \in C} f(z, g(z))=0 .
$$

Letting $x=\left(y_{1}, y_{2}, \ldots, x_{i}, \ldots y_{n}\right)(i=1, \ldots, n)$ in the last inequality we conclude that

$$
\left.f_{i}(y)-f_{i}\left(y_{1}, \ldots, g\left(x_{i}\right), \ldots, y_{n}\right)\right) \leq 0
$$

for every $x_{i} \in C_{i}, i=1, \ldots, n$.

The following result due to M. Sion [5] has important applications in convex analysis and games theory:

Theorem 9. Let $D$ and $F$ be two nonempty, compact and convex subsets and let $f: D \times F \rightarrow \mathbb{R}_{+}$be a function which is upper-semicontinuous and quasi-concave function in the first variable, and lower-semicontinuous and quasi-convex in the second variable. Then

$$
\min _{y \in F} \max _{x \in D} f(x, y)=\max _{x \in D} \min _{y \in F} f(x, y) .
$$

\footnotetext{
${ }^{1}$ Corrected, February 12, 2009. The authors thank Professor S. Park for calling their attention to a mistake in the original version of this theorem.
} 
This result can be derived via Theorems 5 and 7 above provided that

$$
\inf _{z \in D} f\left(z, g_{1}(z)\right)=\sup _{z \in D} f\left(z, g_{2}(z)\right) .
$$

for suitably chosen continuous onto functions $g_{1}$ and $g_{2}$. In fact, the hypothesis of Sion's theorem make possible to apply Theorems 5 and 7 and thus

$$
\inf _{z \in D} f\left(z, g_{1}(z)\right) \leq \max _{x \in D} \min _{y \in F} f(x, y) \leq \min _{y \in F} \max _{x \in D} f(x, y) \leq \sup _{z \in D} f\left(z, g_{2}(z)\right),
$$

for all continuous onto functions $g_{1}, g_{2}: D \rightarrow F$. While the topological condition (4.1) can be easily verified in a number of particular cases, we do not know how general is it. Results of this type, concerning the existence of continuous onto maps relating compact convex sets, may be found in [2].

Acknowledgement 1. Paper supported by Grant CNCSIS 80/2006.

\section{REFERENCES}

[1] J. M. Borwein and A. S. Lewis, Convex Analysis and Nonlinear Optimization, Springer-Verlag, Berlin, 2000.

[2] J. G. Hocking and G. S. Young, Topology, Dover, 1988.

[3] J. Kindler, Über Spiele auf konvexen Mengen, in Operations Res. Vehrfahren, Hain, Meisenheim/Glan, (1977), 695-704.

[4] J. Kindler, A Simple proof of Sion's Minimax Theorem, The American Mathematical Monthly, 112 (2005), 356-358.

[5] M. Sion, On general minimax theorems, Pacific J. Math, 8 (1958), 171-176.

Department of Mathematics, University of Craiova, Craiova RO-200585, Roman

E-mail address: cniculescu@central.ucv.ro

Department of Mathematics, University of Craiova, Craiova RO-200585, Romania

E-mail address: roventaionel@yahoo.com 